\title{
Towards a network of observatories in terrestrial environmental research
}

\author{
H. Bogena ${ }^{1}$, K. Schulz ${ }^{2}$, and H. Vereecken ${ }^{1}$ \\ ${ }^{1}$ Agrosphere Institute (ICG-IV), Institute of Chemistry and Dynamics of the Geosphere (ICG), Research Centre Juelich, \\ 52425 Juelich, Germany \\ ${ }^{2}$ Computational Environmental Systems, UFZ - Centre for Environmental Research Leipzig-Halle, 04318 Leipzig, Germany
}

Received: 23 January 2006 - Revised: 22 May 2006 - Accepted: 3 July 2006 - Published: 26 September 2006

\begin{abstract}
In order to address the challenges of global change, interdisciplinary research in terrestrial environmental science is of great importance. Several environmental research networks have already been established in order to monitor, analyse and predict the impact of global change on different compartments and/or matter cycles of the environment. Typically these environmental research networks have focused on specific research questions, and compartments, such as CarboEurope, FLUXNET and ILTER. In this paper, we propose the establishment of a network of terrestrial observatories, defined as a system consisting of the subsurface environment, the land surface including the biosphere, the lower atmosphere and the anthroposphere. Hydrological units will be used as the basic scaling units in a hierarchy of evolving scales and structures ranging from the local scale to the regional scale for multi-disciplinary process studies. Although terrestrial systems are extremely complex, the terrestrial component in most process-based climate and biosphere models is typically represented in a very conceptual and often rudimentary way. Remedying this deficiency is therefore one of the most important challenges in environmental and terrestrial research, and we suggest that terrestrial observatories could be an important step towards a new quality in environmental and terrestrial research.
\end{abstract}

\section{Introduction}

In the course of the 20th century, the activities of mankind and their consequences extended over the whole planet Earth putting at risk present resources for life and jeopardizing the development possibilities of future generations (e.g. EEA, 2004; McCarthy et al., 2001). The intensity of these anthropogenic interferences affects the global biogeochemical cy-

Correspondence to: H. Bogena

(h.bogena@fz-juelich.de) cle in a dramatic way. Atmospheric trace gases and aerosols control the quality of our air and have a great impact on the radiation budget of the atmosphere. The composition and distribution of these substances in space and time contribute in a manner still not fully understood to regional and global climate changes. Land use and land cover changes as a consequence of new EU agricultural policies or the extended sealing of urban areas will have a significant impact on biodiversity and the buffering capacities of soil systems. Chemicals introduced into the environment will also alter the functioning of ecosystems and affect water and food quality.

In order to evaluate the effects of regional and global natural and anthropogenic changes and to develop technological, political and economic instruments for control and regulation, a comprehensive knowledge of the environmental systems, processes and feedbacks is required. In order to address theses challenges, interdisciplinary research in terrestrial environmental science is of great importance. In the sense of sustainable development, the focus must not only be on short-term applications, but the time lags of environmental processes must also be taken into account that only lead to serious impacts on human society in the course of decades.

The term global change is used to encompass these multiple environmental and ecological changes. Global change effects are expected to occur on a medium- (10 years) to long-term scale (100 years and more). Global change is observed in all compartments of the Earth's system, e.g. the increased concentration of $\mathrm{CO}_{2}$ and other trace gases in the atmosphere, global warming, changing weather patterns, the loss of ozone in the stratosphere, land system changes caused by social, demographic and economic forces, and the effects on ecosystems (e.g. deforestation, species extinction, and neophytes) (GLP, 2005).

The scientific community has responded to these environmental changes by performing dedicated experiments (e.g. Knorr et al., 2005) and by establishing environmental

Published by Copernicus GmbH on behalf of the European Geosciences Union. 
research networks to monitor, analyse and predict the impact of global change on different compartments and/or matter cycles of the Earths' environment. Typically these environmental research networks have focused on specific research questions, and compartments, such as CarboEurope (http://www. carboeurope.org/), FLUXNET (http://www.fluxnet.ornl.gov/ fluxnet/index.cfm) or ILTER (http://www.ilternet.edu/). CarboEurope and FLUXNET are coordinating regional observations of exchanges of carbon dioxide, water vapour, and energy between terrestrial ecosystems and the atmosphere. ILTER consists of networks of scientists engaged in long-term, site-based ecological and socioeconomic research.

\section{On the need for a network of terrestrial observatories}

Recent publications demand a stronger integration of monitoring, modelling and regionalisation activities (Parr et al., 2003) as well as an increased realisation of controlled largescale experiments (Osmond et al., 2004). The combination of these research activities requires a new research approach that is based on a clear definition of scales (spatial and temporal) in the systems under study and the way these scales are organised. Therefore it is inevitable that the research activities should be embedded in a geographically distributed infrastructure.

In general, the experimental and modelling activities of the existing research networks have not yet considered the concept of evolving scales and regionalisation issues in an appropriate way. A recent report from the ILTER coordinating committee stated that more emphasis should be placed on putting the results and process-level studies into larger spatial contexts and on using process models as tools for extending field experiments to a wider range of conditions and to predict the causes of environmental changes (Blair, 2004).

Only recently the CarboEurope network has introduced a key innovative activity that will allow the terrestrial carbon balance to be quantified at different scales and with known, acceptable uncertainties. To obtain this goal, the spatial and temporal resolution of the observational and modelling program will be increased in order to arrive at a consistent application of a multiple constraint approach of bottom-up and top-down estimates to determine the terrestrial carbon balance of Europe with the geographical patterns and variability of sources and sinks.

The various systems are adapting at different rates to global changes, since the buffering capacities of the compartments are diverse, e.g. soils are able to buffer pollutants more effectively and over longer times than the atmosphere as the result of higher quantities of reactive interfaces. So far, the existing research networks have focused strongly on specific compartments of terrestrial systems, such as land surface, various parts of the atmosphere, and natural ecosystems, without considering their different buffer capacities. Although there is a growing awareness of subsurface ter- restrial systems being affected by global change and the direct impact of human activity, less attention has been paid to these compartments. Typically measurements of terrestrial data have been used within the existing research networks to support or underpin findings made in other compartments (e.g. CarboEurope or ILTER). A comprehensive and in-depth analysis including the feedback mechanisms to explain and predict, for example, the observed decrease in soil organic matter, the expected changes in the soil moisture regime, evapotranspiration, groundwater recharge and runoff generation, in a nested hierarchy of scales has not been the major focus of these networks. At present, the research infrastructure for addressing these scientific issues is still lacking.

In addition to the impact of global change, which operates in an indirect and global manner, terrestrial systems are strongly impacted by local and direct human activities such as land use changes, opencast mining, road and building constructions, and deforestation. All these activities cause dramatic changes in the terrestrial systems which are not yet monitored in an adequate scientific way.

The increasing anthropogenic interference in the terrestrial system and the changes involved require a multivalent and longer-term utilisation of research areas at the regional scale with corresponding personnel and financial expenditure. $\mathrm{Nu}-$ merous experiments (e.g. summarised in Giardina and Ryan, 2000) have been conducted in order to evaluate the effects of climate change on soil carbon dynamics. However, these experiments rarely continue for more than a few years and thus never provide information on the response of the large, slower pools that will dominate feedback from the soil to the atmosphere over timescales of decades or more (Powlson, 2005). The effects of climate change are much more complex than just an increase of a single parameter, since compensation and acclimatisation processes may occur (Davidson et al., 2000).

A recent study on the long-term behaviour of soil organic matter in UK soils has demonstrated that there has been an observable decrease over the last twenty years (Bellamy et al., 2005). This loss of soil organic matter is attributed to an increase in temperature leading to a stronger degradation of organic matter. It is, however, practically impossible to verify and analyse these findings with mathematical models, because no data are available for parameterisation of the soilplant-atmosphere system and the characterisation of boundary conditions. The salient question of whether this decrease is caused by global change or changes in farming practices can therefore not be corroborated by existing knowledge. At present there are no appropriate measurement platforms to register and observe these changes or the feedback between the soil-vegetation-atmosphere compartments for appropriate timescales.

Since anthropogenic changes of terrestrial systems are mostly a result of societal and economic developments (e.g. demographic evolution, legacy, land use planning etc.), it is necessary to include possible future changes in social and 
economic boundary conditions. Those questions have not been addressed in the existing networks.

The wide range of environmental research initiatives in Germany are of outstanding quality. However, the German research communities, scientific and politically driven ones, suffer from deficiencies in integration and harmonisation. Apart from the long established meteorological networks, Germany still lags behind with respect to worldwide ongoing initiatives of coordinating terrestrial research activities and the establishment of observatories. As an example, the establishment of an LTER Network and sites in Germany (http://www.lter-d.de/) was first discussed in 2004 and is even now only in the initial phase of justification.

To meet these demands, the environmental scientific community has recognized the need to establish terrestrial observatories (TO) in different fields of research such as the ecologically orientated NEON initiative (http://www.neoninc. org/) in order to put results and process level in a larger spatial context. Such observatories are considered as research instruments that consist of a geographically distributed infrastructure, which is networked via state-of-theart communications. Similar initiatives has been taken by the hydrological community (CUAHSI, http://www.cuahsi. org/index.html) and the environmental engineering community (CLEANER, http://http://cleaner.ncsa.uiuc.edu/people/) in the USA to bring forward hydrological research by establishing long-term hydrological observatories having a defined regional representation.

In the framework of the European research network CarboEurope, test sites have been selected for detailed carbon budget analysis (Fig. 1). These sites are equipped with numerous high-quality environmental sensors, e.g. climate stations, eddy covariance towers, soil respiration chambers, soil moisture probes etc., for monitoring the carbon stocks and $\mathrm{CO}_{2}$ fluxes in all relevant compartments and assembling essential state variables and parameters for modelling purposes. From Fig. 1 it becomes apparent that the sites are concentrated in the centre and in the far south of Germany. Also natural, mostly forested, sites are dominant within current observatory initiatives and an extension to anthropogenically impacted, agricultural sites is required - a mission that could be associated ideally with the proposed TO.

\section{Implementation of terrestrial observatories}

A terrestrial system in this context is defined as a system consisting of the subsurface environment, the land surface including the biosphere (organised in ecosystems), the lower atmosphere and the anthroposphere. These systems are organised along a hierarchy of evolving scales and structures ranging from the local scale to the regional scale (Fig. 2). Hydrological units will be used as the basic scaling units, because catchments are bordered by water divides representing laterally well-defined system boundaries.

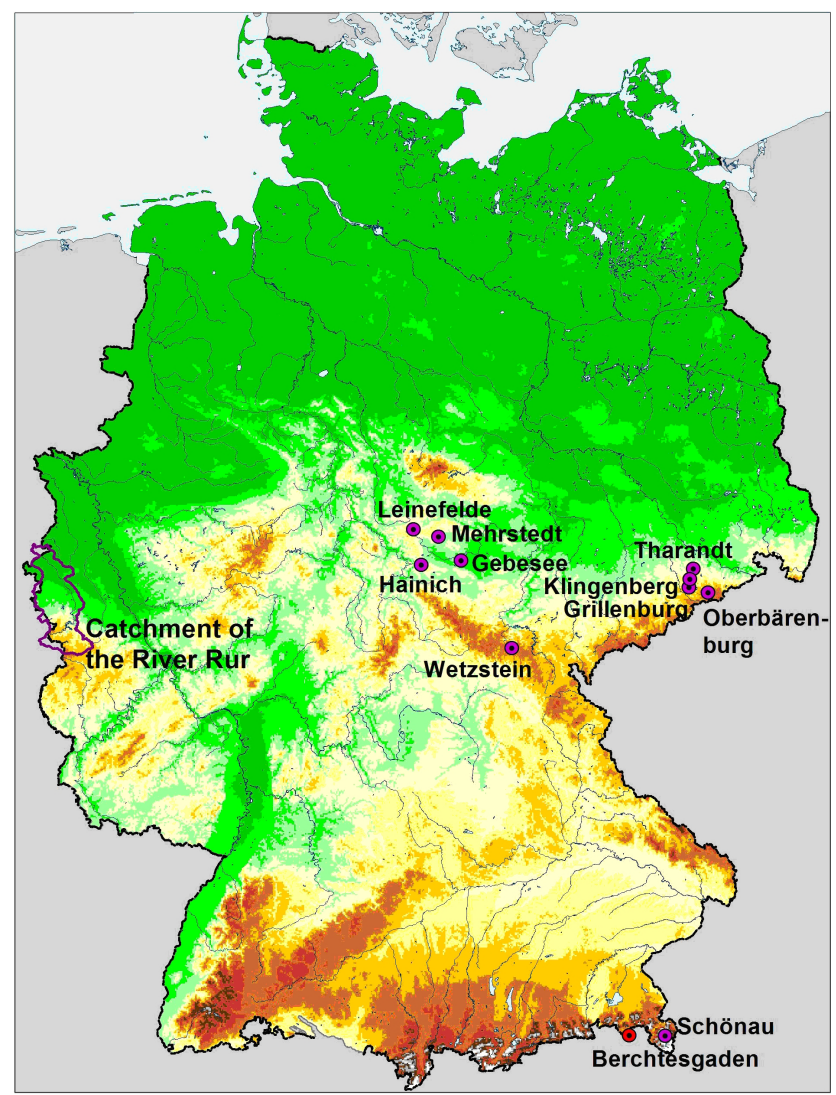

Fig. 1. CarboEurope test sites in Germany and the catchment of the River Rur.

In the framework of a TO, real-time measurement platforms have to be established that will allow terrestrial systems directly influenced by human activities to be observed and enables the development of warning systems. Furthermore, scientific experiments have to be carried out across a nested hierarchy of scales ranging from the local scale (small test sites) to large catchments.

In particular the establishment of terrestrial observatories will contribute to:

- studying the impact of land use changes, climate change, socio-economic developments and human interventions on the evolution of terrestrial systems and their functioning and to analyse the interactions and feedback between the soil-vegetation and atmosphere compartments in these systems across a variety of scales;

- developing methods for upscaling parameters, fluxes and state variables (PFS) that describe processes controlling matter fluxes in soil-plant-atmosphere systems representative of the functioning of the systems at the selected scales based on theoretical and modelling concepts (Fig. 2). This requires the measurement and observation of multi-spatial and multi-temporal distributions 


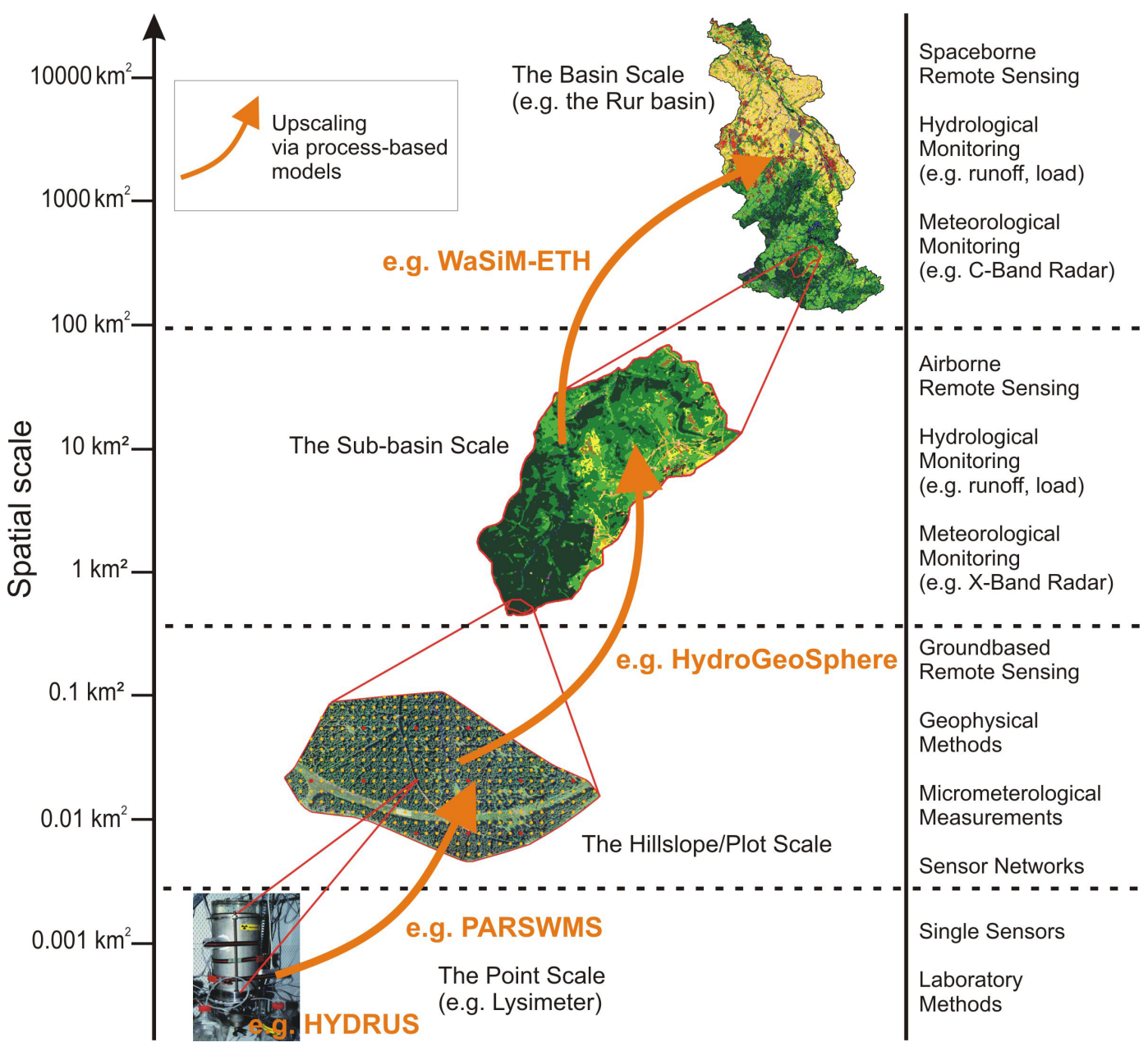

Fig. 2. Hierarchy of evolving scales ranging from the local (point) scale to the regional (basin) scale, associated measurement techniques and model-based upscaling.

of PFS in these systems. Especially the spatial variation of PFS at different scales has not been taken into account in most of the operational networks up to now;

- providing high-quality data to validate existing and newly developed model concepts (e.g. inverse modelling, stochastic data fusion approaches) and upscaling theories to estimate effective parameters, fluxes and state variables at various scales;

- bridging the gap between scales, measurements and modelling currently present in hydrological and terrestrial sciences and developing and improving decision support systems for environmental management from the perspective of sustainable development;

- improving continuously integrated models that predict the evolution of "man-made" terrestrial systems;

- promoting and supporting the development and use of early warning systems (flooding, freshwater quality etc.);
- bringing together different disciplines to create synergetic effects, which will enable the analysis of interactions between natural patterns and processes of landscapes with anthropogenic patterns and processes on different scales.

\section{Criteria for terrestrial observatories}

In order to capture terrestrial-atmospheric feedback, socioeconomical disparities and demographical gradients the spatial scale of a terrestrial observatory should be in the range between $2000 \mathrm{~km}^{2}$ and $10000 \mathrm{~km}^{2}$. Since hydrologic processes exert a fundamental control on aquatic and terrestrial metabolism and nutrient cycling, catchments represent an ideal fundamental unit of TO. A distinct variety in climate, land use, demography etc. should be present over the whole catchment area to enable the consideration of multiple site conditions. By combining different observatories within a network, even larger scale atmospheric feedbacks 
and impacts and thus a more pronounced general link to the atmospheric research community could be established.

Near-natural zones (preferably national parks) should be part of the TO as reference sites. Comparative monitoring strategies that take into account the surrounding landscapes will enable the analysis of long-term environmental changes due to human impact. Ideally, the TO region should be the subject of prospective changes (e.g. open mining, renaturation arrangements, deforestation, economic and demographical developments etc.) in order to analyse the effects of regional changes and disturbances.

Installations for the measurement of environmental and socio-economic quantities, influencing variables and indicators should be available for subsequent integration into the TO network. Within the terrestrial observatory, small-scale research facilities and test areas have to be in place in order to perform detailed process studies. By developing a concept of hierarchical scales this detailed information will be transferred to the regional scale (Fig. 2).

The TO has to be equipped with research instruments designed for long-term measurements. Important parameters, fluxes and state variables (PFS) for the compartments of the atmosphere, hydrosphere, soil, hydrogeology, and biosphere, as well as for socio-economic and demographic problems have to be monitored simultaneously. Different strategies should be considered depending on the time scale of expected changes. Rapid, short-term variations will need to be monitored by fixed installed instruments that have to be networked via state-of-the-art communications in order to enable near-real-time measurements and to simplify data management, e.g. smart sensor networks. Smart sensor networks consist of a multitude of small sensors nodes embedded in the environment and are able to observe phenomena, e.g. temperature or soil moisture fields, with high temporal and spatial resolution. Slower variations of system states might best be observed by a system of mobile and flexible sensor networks that will operate periodically on a regular or eventdriven basis thus allowing a more efficient and wider-area analysis. The monitoring and scientific programme has to be accompanied by high-performance systems for data storage and processing.

\section{Prototype of a terrestrial observatory}

Up to now it has never been attempted to set up multidisciplinary observatories of this spatial scale and complexity. This chapter aims to illustrate the concept of TO by choosing the catchment area of the River Rur as a concrete example to serve as a hydrology-related prototype for terrestrial observatories. It covers a total area of $2354 \mathrm{~km}^{2}$ and is situated in Western Germany (see Fig. 1). The catchment area exhibits a distinct land use gradient: The lowland region in the northern part is characterised by urbanisation and intensive agriculture whereas the low mountain range in the southern part is sparsely populated and includes several drinking water reservoirs. Furthermore, the Eifel National Park is situated in the southern part of the Rur catchment serving as a reference site. In the following, a monitoring concept for the Rur catchment is described that is capable of measuring the spatial-temporal variability of the main hydrological processes and interactions as well as the varying residence times of the terrestrial water stores.

Precipitation radars are considered suitable for estimating rates and distributions of precipitation fields on a range of scales by relating the reflection intensity $\mathrm{Z}$ to the precipitation intensity $R$. A network of point measurements of rain rates can be used in order to inversely establish the Z-R relation. The net ecosystem flux of $\mathrm{H}_{2} \mathrm{O}$, momentum, sensible and latent heat can be measured using eddy covariance systems, which will be installed across important gradients within the TO. These measurements will be co-located with radiation component budget measurements and standard meteorological variables within stream-gauged sub-catchment areas.

Intensive soil moisture monitoring sites have to be collocated to climate towers for coordinated observation. Measurements will be made at several depths with TDR, FD, and $\mathrm{pF}$ sensors into the soil profile, where feasible as far as the water table. In addition to the multi-depth soil moisture monitoring sites, wireless sensor networks have to be established at the sub-catchment scale. The wireless sensor network technology has the potential to reveal dynamic changes in soil moisture with a high spatial-temporal resolution. Ground penetrating radar techniques should be applied for soil water content determination at the intermediate scale. The integrated measuring of water and heat fluxes and soil moisture allows the local closure of the water and energy budget at the plot scale. Such intensive test sites have to be placed along a transect across the TO in representative land cover, soil, and geologic settings to evaluate climate variability on recharge.

In order to obtain spatially distributed information about river discharge rates, the $\mathrm{TO}$ needs to be partitioned into a nested set of sub-catchments that will span distinct assemblages of hydrologic features and several orders of magnitude in drainage area. These sub-catchments have to be instrumented with flow velocity and water level measurement devices for a non-invasive, continuous and very precise monitoring of discharge rates and water quality in real time. More detailed measurements and characterisation of smaller, focal catchments have to be embedded within progressively larger catchments, allowing the critical evaluation and development of hydrologic scaling strategies. Additionally, for the analysis of the groundwater flow system and groundwater exchange rates on a regional scale the monitoring of natural tracers (e.g. radon) of ground and surface waters within the TO should be carried out.

The evaluation of the effects of global and regional changes on the hydrological regime in the Rur catchment have to be accompanied by an analysis of the consumptive 
water use and water management activities as well as point source emissions (e.g. water reservoirs, draining of mines, discharges from industrial and municipal sewage treatment etc.).

In order to monitor the processes controlling matter fluxes in soil-plant-atmosphere systems the described hydrological monitoring concept has to be extended by adding other relevant compartments (e.g. soil, vegetation, and atmosphere) and by a socioeconomic analysis. This may include the identification of socioeconomic forces that drive the intensification and/or de-intensification processes (e.g. demographic and technological change) and the feedback to the ecological system (e.g. on the state of soil, water and air quality) as well as the analysis of ecological impacts of land use changes across and within sectors (e.g. from agricultural to industrial types or from chemical to energy production).

\section{Conclusions}

Environmental and terrestrial research still has to deal with a gap between the scale at which we understand the functioning of terrestrial systems and the scale at which management strategies for our environment have to be developed and applied. There are several reasons for this:

- Compared to the atmospheric or the marine compartments, the terrestrial compartment is a highly complex and diverse system, exhibiting strong heterogeneities across all scales.

- Terrestrial systems have an inherent spatial variability characterised by continuously evolving scales and a multitude of processes interacting in a complex manner within and across compartments of terrestrial systems.

- The prediction and description of these processes at larger scales is hampered by the lack of validated models caused by the non-availability of appropriate measurement technologies and scientifically based concepts and approaches to parameterise these models.

- In this respect, terrestrial research lags some way behind atmospheric and marine sciences both in terms of operational measurement platforms and modelling.

- Terrestrial observatories present ideal locations for the development and the validation of integrated modelling approaches at the regional scale.

Despite its complexity, the terrestrial component in most process-based climate and biosphere models is typically represented in a very conceptual way. Against the background of global change, remedying this deficiency is therefore one of the most important challenges in environmental and terrestrial research. Therefore, the implementation of terrestrial observatories could be an important step towards a new quality of environmental and terrestrial research.
Edited by: R. Barthel, J. Götzinger, G. Hartmann, J. Jagelke, V. Rojanschi, and J. Wolf

Reviewed by: anonymous referees

\section{References}

Bellamy, P., Loveland, P., Bradley, R., Lark, R., and Kirk, G.: Carbon losses from all soils across England and Wales 1978-2003, Nature, 437, 245-248, 2005.

Blair, J.: Meeting Minutes of the Spring 2004 LTER Coordinating Committee (CC) Meeting, 28-29 April 2004, Santa Barbara, CA, 2004.

CarboEurope: http://www.carboeurope.org/, date of access: 7 June 2006.

Collaborative Large-Scale Engineering Analysis Network for Environmental Research (CLEANER): http://cleaner.ncsa.uiuc.edu/ people/, date of access: 7 June 2006.

Consortium of Universities for the Advancement of Hydrologic Science (CUAHSI): http://www.cuahsi.org/index.html, date of access: 7 June 2006.

Davidson, E. A., Trumbore, S. E., and Amundson, R.: Soil warming and organic carbon content, Nature, 408, 789-790, 2000.

European Environmental Agency (EEA): Impacts of Europe's changing climate - an indicator-based assessment, EEA Report, No. 2/2004, 2004.

FLUXNET: http://www.fluxnet.ornl.gov/fluxnet/index.cfm, date of access: 7 June 2006.

Giardina, C. P. and Ryan, M. G.: Evidence that decomposition rates of organic carbon in mineral soil do not vary with temperature, Nature, 404, 858-861, 2000.

Global Land Project (GLP): Science Plan and Implementation Strategy, IGBP Report No. 53/IHDP Report No. 19, 2005.

International Long Term Ecological Research - Deutschland (LTER-D): http://www.lter-d.de/, date of access: 7 June 2006.

Knorr, W., Prentice, I. C., House, J. I., and Holland, E. A.: Longterm sensitivity of soil carbon turnover to warming, Nature, 433, 298-301, 2005.

McCarthy, J. J., Canziani, O. F., Leary, N. A., Dokken, D. J., and White, K. S.: Climate Change 2001: Impacts, Adaptation, and Vulnerability, IPCC Third Assessment Report, Cambridge University Press, 2001.

National Ecological Observatory Network initiative (NEON): http: //www.neoninc.org/, date of access: 7 June 2006.

International Long Term Ecological Research (ILTER): http://www. ilternet.edu/, date of access: 7 June 2006.

Osmond, B., Ananyev, G., Berry, J., Langdon, C., Kolber, Z., Lin, G., Monson, R., Nichol, C., Rascher, U., Schurr, U., Smith, S., and Yakir, D.: Changing the way we think about global change research: scaling up in experimental ecosystem science, Global Change Biology, 10, 393-407, 2004.

Parr, T. W., Sier, A. R. J., Battarbee, R. W., Mackay, A., and Burgess, J.: Detecting environmental change: science and society-perspectives on long-term research and monitoring in the 21st century, The Science of the Total Environment, 310, 1-8, 2003.

Powlson, D.: Will soil amplify climate change? Nature, 433, 204205, 2005. 